\title{
CITOGENETICA DE HIBRIDOS ENTRE TURNERA GRANDIDENTATA (4x) Y T. SUBULATA Y T. SCABRA (2x) (TURNERACEAE)
}

por AVELIANO FERNANDEZ' y MARIA M. ARBO ${ }^{2}$

\section{Summary}

Turnera subulata and T. scabra, $2 \mathrm{n}=2 \mathrm{x}=10$, were crossed with $T$. grandidentata, $2 \mathrm{n}=4 \mathrm{x}=20$, and the hybrids obtained were studied cytologically to determine the relationship between these species. All the hybrids had $2 \mathrm{n}=3 \mathrm{x}=15$ and irregular meiosis. T. subulata $\mathrm{x} T$. grandidentata had a mean chromosome association of 4.28 univalents, 4.16 bivalents and 0,73 trivalents. $T$. scabra $\times$ T. grandidentata had a mean chromosome association of 4.53 univalents, 4,42 bivalents, 0.53 trivalents and 0.03 cuadrivalents. The cytogenetic study of these hybrids indicates that these three species have the same basic genome.

The genomic formulas $\mathrm{A}^{\mathrm{su}} \mathrm{A}^{\mathrm{su}}$ for T. subulata, $\mathrm{A}^{\mathrm{sc}} \mathrm{A}^{\mathrm{sc}}$ for T. scabra and $\mathrm{A}^{g} \mathrm{~A}^{\mathrm{g}} \mathrm{A}^{\mathrm{r}} \mathrm{A}^{r}$ for $T$. grandidentata were proposed in previous papers. The associations and configurations found in the hybrids analyzed in this paper support the genomic formulas proposed.

\section{Introducción}

Entre las especies de Turnera L. de la serie Canaligerae Urban se realizaron numerosos cruzamientos y se obtuvieron varios híbridos (Arbo y Fernández, 1987). El objetivo del presente trabajo es analizar el comportamiento meiótico de los híbridos entre las tres especies siguientes: $T$.

Facultad de Ciencias Exactas y Naturales y Agrimensura (UNNE).

Facultad de Ciencias Agrarias (UNNE).

Miembros de la Carrera del Investigador Científico (CONICET). Instituto de Botánica del Nordeste, C.C. 209,3400 Corrientes. 
grandidentata (Urban) Arbo, $2 \mathrm{n}=4 \mathrm{x}=20, T$. subulata $S \mathrm{~m} ., 2 \mathrm{n}=2 \mathrm{x}=10$ y $T$. scabra Millsp., $2 \mathrm{n}=2 \mathrm{x}=10$. Estas dos últimas especies poseen también citotipo tetraploide, $2 n=4 x=20$ (Fernández 1987).

T. grandidentata fue considerada como un alotetraploide típico (Fernández, 1987), pero posteriormente se demostró que es un alotetraploide segmentario (Fernández y Arbo, 1990). El híbrido entre los citotipos diploides de T. subulata y T. scabra fue estudiado previamente (Fernández y Arbo, 1989).

También se analizan la fertilidad del polen y los caracteres morfológicos de los progenitores y de los híbridos.

\section{Material y métodos}

Se enumeran a continuación las accesiones utilizadas como progenitoras así como los híbridos obtenidos, cuyos testigos se conservan en el herbario del Instituto de Botánica del Nordeste (CTES).

T. grandidentata, $2 \mathrm{n}=4 \mathrm{x}=20$, Paraguay, Central, Itá Enramada, Arbo 1674 (Grl); Paraguay, Cordillera, Col. Tobaty, Schinini 23981 (Gr4).

T. scabra, $2 \mathrm{n}=2 \mathrm{x}=10$, Nicaragua, Managua, ca. Mateare, Araquistain 1354 (I2).

T. subulata, $2 \mathrm{n}=2 \mathrm{x}=10$, Brasil, Maranhao, São Luis, Arbo 2410 (E2).

T. grandidentata $(\mathrm{Gr} 1) \times T$. scabra (I2), $2 \mathrm{n}=3 \mathrm{x}=15$, Arbo \& Fernández 2723. T. scabra (I2) x $T$. grandidentata $(\mathrm{Gr} 1), 2 n=3 x=15$, Arbo 2574.

T. grandidentata $(\mathrm{Gr} 4) \times T$. subulata $(\mathrm{E} 2), 2 \mathrm{n}=3 \mathrm{x}=15$, Arbo \& Fernández 2736, 2738 y 2748. T. subulata (E2) x T. grandidentata (Gr1), $2 \mathrm{n}=3 \mathrm{x}=15$, Arbo y Fernández 2565.

El procedimiento usado para los cruzamientos está descripto en Arbo y Fernández (1987). Las preparaciones para el análisis meiótico se hicieron fijando los botones florales con etanol absoluto y ácido láctico (5:1) y coloreando con Feulgen. Para mitosis se siguió el mismo procedimiento previo pretratamiento de los ápices radicales con 8 -oxiquinoleína $(0,002 \mathrm{M})$ durante tres horas a temperatura de laboratorio. Para determinar el porcentaje de fertilidad se coloreó el polen con carmín-glicerina, contando no 
menos de 300 granos por flor. El tamaño de los mismos se determinó midiendo no menos de 100 de los granos que se colorearon en los progenitores y a veces menos en los híbridos.

\section{Resultados}

\section{Meiosis}

Las especies progenitoras presentaron meiosis regular, $T$. subulata y T. scabra, $2 \mathrm{n}=2 \mathrm{x}=10$, formaron 5 II y $T$. grandidentata, $2 \mathrm{n}=4 \mathrm{x}=20$ con 10 II (Fernández, 1987).

$T$. subulata $\times T$. grandidentata, $2 \mathrm{n}=3 \mathrm{x}=15$. Estos híbridos presentaron cinco configuraciones diferentes, siendo la más frecuente 5 I + 5 II (Fig. 1) en $53 \%$ de las CMP, seguida por 4 I +4 II +1 III con $27 \%$ (Fig. 2). Se observaron trivalentes en $46 \%$ de las CMP, hasta un máximo de 5 III en una célula (Fig. 3). El promedio de bivalentes por célula es de 4,16, algo menor que el de univalentes, de 4,28 (Tabla 1). En anafase I se observaron cromosomas rezagados (Fig. 4) en $80 \%$ de las CMP, pero en

Tabla 1: Promedio \pm E.S. y variación de las asociaciones cromosómicas en metafase I en híbridos de Turnera

\begin{tabular}{|c|c|c|c|c|c|c|}
\hline Híbrido & $2 n$ & I & II & III & IV & CMP \\
\hline \multicolumn{7}{|l|}{ T. subulata (E2) } \\
\hline $\mathrm{x}$ & 15 & $4,28 \pm 0,13$ & $4,16 \pm 0,08$ & $0,73 \pm 0,07$ & & 152 \\
\hline $\begin{array}{l}\text { T. grandidentata } \\
\text { (Gr1) }\end{array}$ & & $1-5$ & $1-5$ & $0-5$ & & \\
\hline \multicolumn{7}{|l|}{ T. scabra (I2) } \\
\hline $\mathrm{x}$ & 15 & $4,53 \pm 0,15$ & $4,42 \pm 0,13$ & $0,53 \pm 0,13$ & $0,03 \pm 0,003$ & 28 \\
\hline $\begin{array}{l}\text { T. grandidentata } \\
(\mathrm{Gr} 1)\end{array}$ & & $2-7$ & $2-5$ & $0-3$ & $0-1$ & \\
\hline
\end{tabular}


interfase, en P II y/o metafase II el $62 \%$ de las CMP carecía de micronúcleos. Estos resultados estarían indicando que en la mayoría de las células los cromosomas rezagados llegan a los polos telofásicos. En algunas células de anafase I los univalentes se dividen ecuacionalmente (Fig. 4). También se observaron puentes y fragmentos en anafase I. En anafase II todas las células presentaban cromosomas rezagados (Fig. 5).

T. scabra $\times$ T. grandidentata, $2 \mathrm{n}=3 \mathrm{x}=15$. Estos híbridos presentaron cuatro configuraciones diferentes, siendo la más frecuente 5 I + 5 II (Fig. 6) en $50 \%$ de las CMP y $4 \mathrm{I}+4$ II +1 III con $42 \%$. Se observaron trivalentes en $50 \%$ de las células, con un máximo de tres trivalentes y un cuadrivalente en una CMP (Fig. 7). Las pocas células analizadas en anafase I presentan cromosomas rezagados (Fig. 8), pero en interfase no se pudo constatar ningún micronúcleo, sugiriendo que todos los cromosomas alcanzaron los polos telofásicos.

El híbrido $T$. subulata $\times$ T scabra, $2 \mathrm{n}=2 \mathrm{x}=10$, en metafase I presentaba todas las células con 5 II y la fertilidad de polen fue de $70 \%$ (Fernández \& Arbo, 1989).

\section{Polen}

En las tres especies consideradas, como en todas las especies heterostilas de Turnera, los granos de polen de flores brevistilas son de mayor tamaño que los de flores longistilas. También en este caso, los granos de polen coloreados de los híbridos presentan una variación de tamaño mayor que la de los progenitores (Tabla 2).

Normalmente los granos son tricolporados, pero en los híbridos se observaron numerosos granos coloreados con cuatro aberturas, incluso al-

Figs. 1-8.- Meiosis. 1-5, T. subulata x T. grandidentata. 1, metafase I, 5 I + 5 II. 2. metafase I, 4 I + 4 II + 1 III (flecha). 3, metafase I, 5 III. 4, anafase I, cromosomas rezagados. 5, anafase II, cromosomas rezagados. 6-8, $T$. scabra $\times$ T. grandidentata. 6, metafase I, 5 I + 5 II. 7, metafase I, 2 I + 3 III + I IV (flecha). 8, anafase I, cromosomas rezagados. 
123
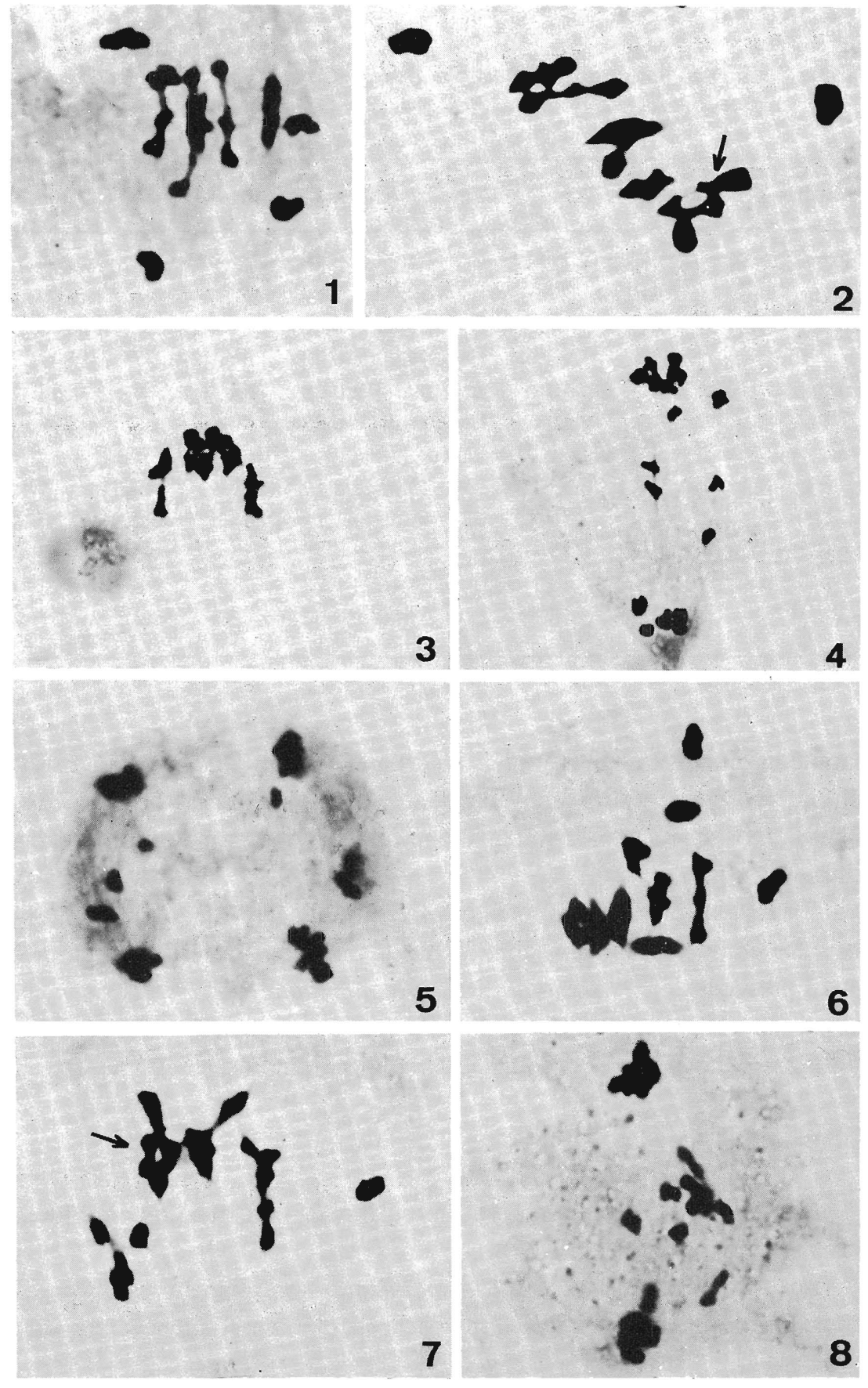
Tabla 2: Tamaño promedio \pm E.S. en $\mu \mathrm{m}$ de los granos de polen coloreados de progenitores e híbridos, $\mathrm{L}=\mathrm{fl}$. longistilas, $\mathrm{B}=\mathrm{fl}$. brevistilas

\begin{tabular}{|c|c|c|c|c|c|c|}
\hline Progenitor o híbrido & $2 n$ & $\mathrm{~L} / \mathrm{B}$ & romedio \pm E.S. & mín. & máx. & ${ }^{\circ}$ granos \\
\hline \multirow{2}{*}{$\begin{array}{r}\text { T. grandidentata }(\mathrm{Gr} 1) \\
(\mathrm{Gr} 4)\end{array}$} & 20 & B & $67,03 \pm 0,40$ & 54 & 78 & 106 \\
\hline & 20 & $\mathrm{~L}$ & $50,46 \pm 0,26$ & 42 & 57 & 100 \\
\hline \multirow[t]{2}{*}{ T. scabra (I2) } & 10 & B & $61,00 \pm 0,20$ & 57 & 72 & 100 \\
\hline & 10 & $\mathrm{~L}$ & $44,00 \pm 0,40$ & 36 & 57 & 100 \\
\hline \multirow[t]{2}{*}{ T. subulata (E2) } & 10 & B & $60,00 \pm 0,18$ & 54 & 64 & 132 \\
\hline & 10 & $\mathrm{~L}$ & $47,00 \pm 0,25$ & 40 & 56 & 124 \\
\hline $\mathrm{Gr} 1 \times 12$ & 15 & $\mathrm{~L}$ & $43,86 \pm 2,19$ & 39 & 49,5 & 4 \\
\hline $\mathrm{I} 2 \times \mathrm{Gr} 1$ & 15 & $\mathrm{~B}$ & $65,52 \pm 1,15$ & 46,5 & 82,5 & 47 \\
\hline \multirow[t]{2}{*}{ Gr4 x E2 } & 15 & $\mathrm{~L}$ & $41,91 \pm 0,60$ & 33 & 55,5 & 61 \\
\hline & 15 & B & $64,10 \pm 0,78$ & 49,5 & 78 & 100 \\
\hline $\mathrm{E} 2 \times \mathrm{Gr} 1$ & 15 & $\mathrm{~L}$ & $63,00 \pm 0,62$ & 46,5 & 72 & 101 \\
\hline
\end{tabular}

gunos con cinco. Entre los granos estériles también hay una gran variación, se ven desde granos muy pequeños, de 12-15 micras de diámetro, inaperturados, hasta granos de tamaño normal; se observan también díadas y tétrades.

En las especies progenitoras el promedio de fertilidad del polen oscila entre 84,06 y $95,50 \%$ (Tabla 3 ). En los híbridos la fertilidad es menor, el promedio en $T$. subulata $\times$ T. grandidentata es de $6,93 \%$ con un máximo de $16 \%$ y un mínimo de $0,86 \%$. En $T$. scabra x $T$. grandidentata fue de $2,91 \%$, con un máximo de $7,6 \%$ y un mínimo de $0,34 \%$.

\section{Morfología}

Las especies progenitoras ya fueron tratadas en trabajos anteriores (Fernández \& Arbo, 1989, 1990).

Los híbridos $T$. subulata $\times$. grandidentata presentan algunas variaciones; el follaje en algunos es verde oscuro, como en $T$. subulata, o ver- 
Tabla 3: Porcentajes de fertilidad de polen en plantas progenitoras e híbridos.

\begin{tabular}{|c|c|c|c|c|c|}
\hline Progenitor o híbrido & $2 n$ & promedio & mínimo & máximo & $\mathrm{n}^{\circ}$ granos \\
\hline \multirow[t]{2}{*}{ T. grandidentata } & 20 & 89,27 & 81,88 & 93 & 2105 \\
\hline & 20 & 92,95 & 86,86 & 96,92 & 3021 \\
\hline T. scabra (12) & 10 & 84,06 & 74 & 95 & 1301 \\
\hline T. subulata $(\mathrm{E} 2)$ & 10 & 95,50 & 92 & 99 & 2306 \\
\hline Gr1 $\times 12$ & 15 & 0,38 & 0,34 & 0,43 & 1036 \\
\hline $\mathrm{I} 2 \times \mathrm{Gr} 1$ & 15 & 4,17 & 1,8 & 7,6 & 2259 \\
\hline $\mathrm{Gr} 4 \times \mathrm{E} 2$ & 15 & 4,75 & 3,4 & 7,15 & 2381 \\
\hline $\mathrm{E} 2 \times \mathrm{Gr} 1$ & 15 & 7,20 & 0,86 & 16 & 5819 \\
\hline
\end{tabular}

de más claro como en $T$. grandidentata, y en uno de ellos (A y F 2748) es variegado; el dentado de las hojas es variable, muy marcado en A y F 2565. El diámetro de la corola oscila entre $3,5-4,8 \mathrm{~cm}$; los pétalos son de color amarillo pálido, más intenso hacia el centro, intermedio entre el amarillo de $T$. subulata y el color marfilino de $T$. grandidentata, y presentan una mancha basal violeta o morada, con borde superior nítidamente definido como en la última especie. Las anteras son anaranjadas, igual que en los progenitores, los estigmas son amarillos, y el número de ramas es intermedio entre los progenitores, pero se parecen más a los de $T$. subulata, que son multífidos. Uno de los híbridos produjo algunos frutos pequeños, con 4-5 semillas, algunas vanas y sólo 1-2 llenas.

Los híbridos T. scabra $\times$ T. grandidentata presentan follaje verde, con hojas marcadamente dentadas. Las flores presentan un diámetro ligeramente mayor que el de los progenitores; los pétalos son de color amarillo pálido, intermedio entre el amarillo intenso de $T$. scabra y el marfilino de $T$. grandidentata, y también tienen mancha basal violeta o morado muy oscuro, de borde superior bien demarcado. Las anteras son anaranjadas, cortas como en $T$. grandidentata y los estigmas amarillos y multífidos, como en T. scabra. 


\section{Discusión y conclusiones}

En los dos híbridos triploides estudiados, la configuración más frecuente es 5 I +5 II con $53 \%$ en $T$. subulata $\times$ T. grandidentata, y 50\% en $T$. scabra x $T$. grandidentata. También en ambos híbridos se observó alta frecuencia de trivalentes, en $46 \%$ de las céluals en $T$. subulata x $T$. grandidentata y $42 \%$ en $T$. scabra $\times$. grandidentata. A medida que aumenta la cantidad de trivalentes por célula, disminuyen en la misma proporción las cantidades de univalentes y bivalentes, lo que indicaría que los trivalentes se formaron por apareamiento de los que hubiesen sido un bivalente y un univalente, o sea apareamiento autosindético y alosindético al mismo tiempo. Estos resultados estarían señalando que las tres especies poseen genomas básicos comunes.

La presencia de un cuadrivalente en $T$. scabra x $T$. grandidentata, posiblemente se deba a una translocación recíproca entre cromosomas de los progenitores. La baja frecuencia de cuadrivalentes observada podría deberse a que los segmentos translocados sean muy pequeños.

El promedio de univalentes es algo superior en $T$. scabra x $T$. grandidentata que en $T$. subulata $\times$ T. grandidentata (Tabla 1), además se encontraron células hasta con siete univalentes en el primer híbrido, mientras que en $T$. subulata $\times$. grandidentata el máximo de univalentes fue de cinco. Estos datos y la presencia de una célula con 5 III en T. subulata x $T$. grandidentata indican que el genoma de $T$. scabra estaría más diferenciado que el de T.subulata con respecto a los de T. grandidentata. Esta idea concuerda con la fertilidad de polen observada en los dos híbridos; en $T$. scabra x $T$. grandidentata la fertilidad promedio es de $2,91 \%$ mientras que en $T$. subulata $\times$. grandidentata es de $6,93 \%$.

Los caracteres morfológicos de T. subulata y $T$. scabra indican que se trata de especies muy afines, lo que se confirma por la semejanza de sus cariotipos (Solís Neffa y Fernández, 1993). Considerando la morfología y la distribución geográfica de estas especies y el comportamiento meiótico y la fertilidad de polen elevada del híbrido diploide entre ellas, así como las características citogenéticas de los híbridos con las otras especies diploides, se sugirió que probablemente $T$. scabra haya derivado de $T$. 
subulata (Fernández y Arbo, 1989). Las diferencias observadas en los híbridos con $T$. grandidentata apoyan la hipótesis anterior.

En trabajos anteriores se propusieron las fórmulas genómicas $\mathrm{A}^{\text {su }} \mathrm{A}^{\text {su }}$ y $\mathrm{A}^{\mathrm{sc}} \mathrm{A}^{\mathrm{sc}}$ para $T$. subulata y $T$. scabra respectivamente (Fernández y Arbo, 1989) y $\mathrm{A}^{\mathrm{E}} \mathrm{A}^{ \pm} \mathrm{A}^{\mathrm{r}} \mathrm{A}^{\mathrm{r}}$ para $T$. grandidentata (Fernández y Arbo, 1990). Las asociaciones cromosómicas y configuraciones halladas en los híbridos entre estas especies apoyan las fórmulas genómicas propuestas.

\section{Reconocimientos}

Esta investigación ha sido subsidiada con los PID números 511 y 595 del CONICET.

\section{Bibliografía}

Arbo, M.M. \& A. Fernández. 1987. Cruzamientos intra e interespecíficos en Turnera, serie Canaligerae. Bonplandia 6(1): 23-38.

Fernández, A. 1987. Estudios cromosómicos en Turnera y Piriqueta (Turneraceae). Bonplandia 6(1): 1-22.

Fernández, A. \& M.M. Arbo. 1989. Relaciones genómicas entre cuatro especies diploides de Turnera con flores amarillas (serie Canaligerae). Bonplandia 6(2): 93-109.

- 1990. Gametas no reducidas y relaciones genómicas en tres especies de Turnera (Turneraceae). Darwiniana 30(1-4): 21-26.

Solís Neffa, V.G. \& A. Fernández. 1993. Estudios cromosómicos en especies de Turnera (Turneraceae). Bonplandia 7 (1-4): 95-112.

Urban, I. 1883. Monographie der familie der Turneraceen. Jahrb. Königl. Bot. Gart. Berlin 2: 1-152. 